\title{
Thermal-difference states of light: true states of heralded photons
}

\author{
Dmitri Horoshko ${ }^{1,2, *}$, Stephan De Bièvre ${ }^{3}$, Giuseppe Patera ${ }^{1}$, and Mikhail Kolobov ${ }^{1}$ \\ ${ }^{1}$ Univ. Lille, CNRS, UMR 8523 - PhLAM - Physique des Lasers Atomes et Molécules, F-59000 Lille, \\ France \\ ${ }^{2}$ B. I. Stepanov Institute of Physics, NASB, Nezavisimosti Ave. 68, Minsk 220072 Belarus \\ ${ }^{3}$ Univ. Lille, CNRS, UMR 8524 - Laboratoire Paul Painlevé, F-59000 Lille, France
}

\begin{abstract}
We introduce a three-parameter family of single-mode optical states whose density operator is a weighted difference of two thermal states, the thermal-difference states. We identify the parameter values for which these states have a negative non-singular $P$-function, implying they are nonclassical. We show that the states of the "heralded photons" generated via spontaneous parametric downconversion belong to this family, with the three parameters corresponding to the nonlinear gain and the losses in the signal and the idler channels. The thermal-difference states yield new benchmark states for the analysis of nonclassicality and quantum macroscopicity criteria.
\end{abstract}

\section{Introduction}

In the last decades much attention is attracted to the photon heralding technique [1- -9$]$, which consists in generating a photon pair in two modes and detecting a photon of one (idler) mode, preparing thus the other (signal) mode in a single-photon state. In the ideal case, where exactly one photon pair is generated in a given time window and there is no loss, the conditional state of the signal field is a one-photon Fock state. However, in realistic experimental conditions two and more pairs can be generated by the source at a time and the light collection and detection is accompanied by loss and non-unit quantum efficiency of the photodetector. These factors lead to appearance of multiphoton and vacuum components in the state of the signal field. Traditionally, these components are viewed as an undesirable "contamination" of the single-photon state of the signal mode, whose "purity" is measured by the intensity correlation function at zero delay [3-9].

In this work we consider spontaneous parametric downconversion (SPDC) as a source of photon pairs and find the density matrix of the signal mode conditioned by a photon detection in the idler one. We take into account three physical parameters of the scheme: parametric gain of the SPDC process $r$, transmittance of the idler channel $\eta$, which includes the quantum efficiency of the photodetector, and the transmittance of the signal channel $\mu$. We show that the conditional state of the signal mode is a weighted difference of two thermal states. We call such states thermal-difference states and study some of their properties below.

\footnotetext{
*e-mail: Dmitri.Horoshko@univ-lille.fr
} 


\section{Generation of thermal-difference states}

In the process of SPDC an undepleted classical pump wave, passing through a nonlinear crystal, produces signal and idler waves In a typical SPDC scenario these waves are multimode, and a set of Schmidt modes $[10,11]$ can be defined for each wave such that a signal mode is correlated to the corresponding idler mode and the joint state of two photons is entangled. However, for the production of heralded photons a single-mode regime can be realized by tuning the pump pulse spectral width to that of the phase-matching function [0].

Let us denote the signal mode by A and the idler mode by B and ascribe to these modes annihilation operators $a$ and $b$ respectively. The joint state of two modes at the crystal output is $[12]$

$$
|\psi(r)\rangle_{A B}=e^{r\left(a^{\dagger} b^{\dagger}-a b\right)}|0\rangle_{A}|0\rangle_{B}=\operatorname{sech} r \sum_{n=0}^{\infty} \tanh ^{n} r|n\rangle_{A}|n\rangle_{B},
$$

where $r$ is the squeezing parameter [13] determined by the pump amplitude, the nonlinear susceptibility of the crystal and its length.

We are interested in finding the state of the mode A under condition of a click of detector monitoring the mode $\mathrm{B}$ in the general case, where the losses in the idler and signal channels are characterized by their intensity transmittances $\eta$ and $\mu$ respectively. We consider first the simplest case of no loss and unit quantum efficiency of the detector, $\eta=\mu=1$. A detector not resolving the number of photons and having unit quantum efficiency is characterized by the positive-operator valued measure (POVM) consisting of just two operators: operator $\Pi_{\text {off }}^{B}=|0\rangle_{B B}\langle 0|$ corresponding to no click and operator $\Pi_{\text {on }}^{B}=\mathbb{I}_{B}-|0\rangle_{B B}\langle 0|$ corresponding to a click. Here $\mathbb{I}_{B}$ is the identity operator for the mode B. Under condition of observing a click at the detector monitoring the mode $\mathrm{B}$, the conditional (unnormalized) state of the mode $\mathrm{A}$ is

$$
\begin{aligned}
\tilde{\rho}^{A} & =\operatorname{Tr}_{B}\left\{\Pi_{\mathrm{on}}^{B}|\psi(r)\rangle_{A B A B}\langle\psi(r)|\right\}=\operatorname{sech}^{2} r \sum_{n=1}^{\infty} \tanh ^{2 n} r|n\rangle_{A A}\langle n| \\
& =\rho_{\mathrm{th}}\left(q_{0}\right)-\left(1-q_{0}\right)|0\rangle_{A A}\langle 0|,
\end{aligned}
$$

where $q_{0}=\tanh ^{2} r$. Here we have used the definition of thermal state of optical mode $[14]$

$$
\rho_{\mathrm{th}}(q)=(1-q) \sum_{n=0}^{\infty} q^{n}|n\rangle\langle n|,
$$

where $|n\rangle$ is the $n$-photon Fock state, and $q \in[0,1)$ is a parameter, related to the temperature $T$ and the mean photon number $\langle n\rangle$ as

$$
q=e^{-\frac{\hbar \omega}{k_{B} T}}=\frac{\langle n\rangle}{\langle n\rangle+1},
$$

with $\omega$ being the circular frequency of the mode and $k_{B}$ the Boltzmann constant. As shown by Eq. (3), the thermal state is diagonal in the Fock basis and the number of photons follows the geometric distribution. The limit $q \rightarrow 0$ corresponds to zero temperature and zero mean photon number, i.e. to the vacuum state $\rho_{\text {th }}(0)=|0\rangle\langle 0|$. The opposite limit $q \rightarrow 1$ corresponds to infinitely growing temperature. In general, $q$ is a monotonically increasing function of temperature $T$ and can be considered as "alternative temperature".

Upon normalisation of Eq. (2) we obtain for the state of the mode A

$$
\rho^{A}=\frac{\tilde{\rho}^{A}}{\operatorname{Tr}\left\{\tilde{\rho}^{A}\right\}}=\frac{1-q_{0}}{q_{0}}\left(\frac{\rho_{\mathrm{th}}\left(q_{0}\right)}{1-q_{0}}-|0\rangle\langle 0|\right),
$$


which corresponds to the "truncated thermal state" [15] or the "vacuum-removed thermal state" [16]. This state contains no vacuum component, which has a simple physical explanation: in the absence of losses a click in mode B corresponds to the presence of at least one photon in the mode A.

Now we consider a more complicated scenario, where the quantum efficiency of the idler channel $\eta$ can be less than 1, but there are no losses in the signal channel. It can be shown that in this case the POVM of the detector is given by the operator [17]

$$
\tilde{\Pi}_{\mathrm{off}}^{B}=\sum_{n=0}^{\infty}(1-\eta)^{n}|n\rangle_{B B}\langle n|=\frac{\rho_{\mathrm{th}}(1-\eta)}{\eta},
$$

corresponding to no click, and the operator $\tilde{\Pi}_{\text {on }}^{B}=\mathbb{I}_{B}-\tilde{\Pi}_{\text {off }}^{B}$, corresponding to a click. Under condition of observing a click at the detector $\mathrm{B}$, the conditional (unnormalized) state of the mode A reads

$$
\tilde{\rho}^{A}=\operatorname{Tr}_{B}\left\{\tilde{\Pi}_{\mathrm{on}}^{B}|\psi(r)\rangle_{A B A B}\langle\psi(r)|\right\}=\rho_{\mathrm{th}}\left(q_{0}\right)-\frac{1-q_{0}}{1-q_{0}(1-\eta)} \rho_{\mathrm{th}}\left(q_{0}(1-\eta)\right),
$$

which is a weighted difference of two thermal states of mode A.

The final scenario includes losses of the mode A, which are modelled by a beam splitter with intensity transmission $\mu$. When a field in a thermal state $\rho_{\text {th }}\left(q_{0}\right)$ passes through such a beam-splitter and the state of the reflected field is traced out, the state of the transmitted field is a thermal state with a lower temperature $\rho_{\text {th }}(q)$. The value of $q$ can be found from the transformation of the mean photon number $\langle n\rangle=\mu\langle n\rangle_{0}$, where $\langle n\rangle_{0}=q_{0} /\left(1-q_{0}\right)$ and $\langle n\rangle=q /(1-q)$ are mean photon numbers at the input and the output correspondingly. For the output temperature we find with the help of Eq. (4)

$$
q=\frac{\mu\langle n\rangle_{0}}{\mu\langle n\rangle_{0}+1}=\frac{\mu q_{0}}{1-q_{0}(1-\mu)} .
$$

Applying such a transformation to both summands of Eq. (7) and normalizing the resulting state, we arrive after some algebra at the state of the signal mode, which we call "thermaldifference state":

$$
\rho^{(-)}(q, p, \gamma)=\mathcal{N}\left(\frac{\rho_{\mathrm{th}}(q)}{1-q}-\gamma \frac{\rho_{\mathrm{th}}(q p)}{1-q p}\right)=\mathcal{N} \sum_{n=0}^{\infty}\left(q^{n}-\gamma(q p)^{n}\right)|n\rangle\langle n|,
$$

where

$$
\mathcal{N}=\frac{(1-q)(1-q p)}{1-q p-\gamma(1-q)}
$$

is a normalization factor. The parameters $(q, p, \gamma)$ vary from 0 to 1 and are related to the physical parameters $\left(q_{0}, \eta, \mu\right)$, varying in the same limits, by Eq. (\&) and

$$
p=(1-\eta) \gamma=(1-\eta) \frac{1-q_{0}(1-\mu)}{1-q_{0}(1-\eta)(1-\mu)} .
$$

It is easy to see that for $p, \gamma \in[0,1)$ the density operator, defined by ( 9 ), is always positive, since $q^{n}-\gamma(q p)^{n}>0$ for any $n$ if $q \neq 0$ and this operator is the vacuum state if $q=0$.

Thus, we have shown that the states conditionally prepared by measuring the idler mode of a two-mode squeezed light, by the technique known as "photon heralding", belong to the three-parameter family of thermal-difference states. 


\section{Nonclassicality of thermal-difference state}

A remarkable property of the introduced family of states is the simple structure of their quasiprobability distributions. Here we analyze only the Glauber-Sudarshan $P$-function [14], the other quasiprobability distributions will be considered elsewhere. The $P$-function of the thermal difference state reads

$$
P^{(-)}(\alpha \mid q, p, \gamma)=\frac{\mathcal{N}}{q \pi}\left(e^{-|\alpha|^{2}(1-q) / q}-\frac{\gamma}{p} e^{-|\alpha|^{2}(1-q p) / q p}\right) .
$$

This function is shown in Fig. 4 together with the corresponding photon number distribution $\left\langle n\left|\rho^{(-)}\right| n\right\rangle$ for experimentally feasible values of the parameters.
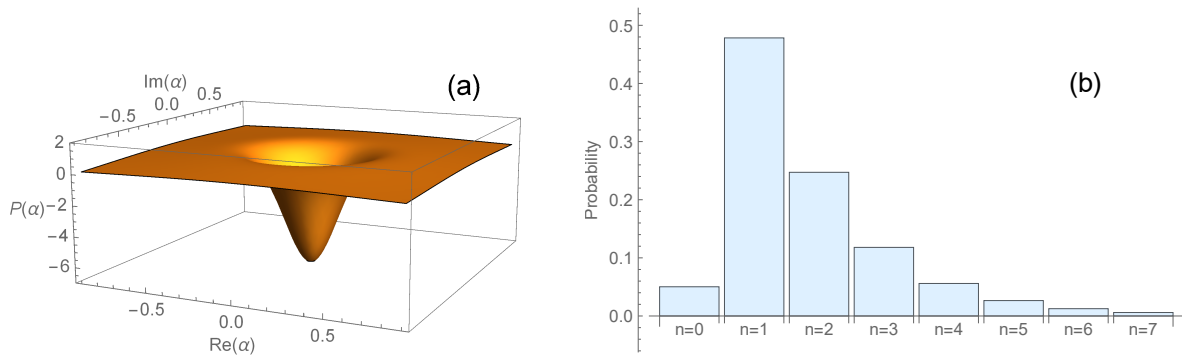

Figure 1. (a) The $P$-function and (b) the photon number distribution for the thermal-difference state generated in a photon heralding experiment with $q_{0}=0.5$ and $\eta=\mu=0.9$. This corresponds to the parameters $(q, p, \gamma)=(0.47,0.1,0.95)$. Negativity of the $P$-function is a signature of nonclassicality of this conditionally prepared state. The vacuum component $(n=0)$ appears in the photon number distribution due to loss in the signal channel $(\mu<1)$.

For sufficiently low values of $\gamma$ the second term in $(12)$ is negligible with respect to the first one and the thermal-difference state approaches the thermal one, having a classical analog. Thus, the non-classical states, which are the most interesting from the point of view of quantum information, are the states with rather high values of $\gamma$. For sufficiently high $\gamma$ the minimal value of $P^{(-)}(\alpha \mid q, p, \gamma)$ is reached at the origin, $\alpha=0$, and this value is

$$
P^{(-)}(0 \mid q, p, \gamma)=\frac{\mathcal{N}}{q \pi}\left(1-\frac{\gamma}{p}\right),
$$

which is negative whenever $\gamma>p$. Note that due to (41) for heralded photons it is always the case if $\eta>0$.

Thus we have shown that the $P$-function of a thermal-difference state for sufficiently high $\gamma$ takes negative values near the origin. The $P$-function of an optical mode, even when it takes negative values, can be reconstructed from a series of measurements. Negative $P$ function of a photon-added thermal state [10] has been successfully reconstructed from the experimental data [19]. It can be shown that the photon-added thermal states belong to the class of thermal-difference states introduced above. As we have shown, these states may be generated by simple photon heralding technique, not requiring thermal seed for the signal as in Ref. [19].

The regularity of the $P$-function and simplicity of the nonclassicality condition, $\gamma>p$, make the introduced family of states an excellent benchmark for nonclassicality tests. There is a number of generic quantum states of a single-mode optical field which are used for testing various nonclassicality measures, the most popular being the Fock state, the squeezed state 
[13] and the Schrödinger cat state, either with two [20-22] or multiple components [23, 24]. Though these states allow one to compare different measures, they are limited to the class of pure states. Generic mixed states include squeezed thermal states, photon-added thermal states [18] and thermalized cat states [25]. The new family of thermal-difference states widens this class significantly. Note that the measures which are quadratic in the density operator can be analytically calculated for the states of this family, because their quasiprobabilities are differences of two Gaussians.

\section{Conclusions}

We have introduced a family of single-mode optical states characterized by non-singular $P$-functions, which can take negative values, and have shown that these states correspond to the states of light, conditionally prepared by the technique of "photon heralding". The states of this family may be classical or nonclassical for different regions of their parameters, which is reflected by the negativity of the $P$-function. It would be interesting to use the states of this family for comparison of several recently proposed quantum macroscopicity and nonclassicality measures for the optical field, like the maximal quadrature quantum Fisher information [20] and the operator ordering sensitivity [27].

\section{Acknowledgments}

This work was supported in part by the Labex CEMPI (ANR-11-LABX-0007-01) and by the Nord-Pas de Calais Regional Council and FEDER through the Contrat de Projets ÉtatRégion (CPER), and in part by the European Union's Horizon 2020 research and innovation programme under grant agreement No 665148 (QCUMbER).

\section{References}

[1] B.Y. Zel'dovich, D.N. Klyshko, JETP Lett. 9, 40 (1969)

[2] C.K. Hong, L. Mandel, Phys. Rev. Lett. 56, 58 (1986)

[3] G. Brida, I.P. Degiovanni, M. Genovese, A. Migdall, F. Piacentini, S.V. Polyakov, I.R. Berchera, Opt. Express 19, 1484 (2011)

[4] M. Förtsch, J.U. Fürst, C. Wittmann, D. Strekalov, A. Aiello, M.V. Chekhova, C. Silberhorn, G. Leuchs, C. Marquardt, Nat. Commun. 4, 1818 (2013)

[5] F. Kaneda, B.G. Christensen, J.J. Wong, H.S. Park, K.T. McCusker, P.G. Kwiat, Optica 2, 1010 (2015)

[6] P. Vergyris, T. Meany, T. Lunghi, G. Sauder, J. Downes, M. Steel, M. Withford, O. Alibart, S. Tanzilli, Sci. Rep. 6, 35975 (2016)

[7] J.B. Spring, P.L. Mennea, B.J. Metcalf, P.C. Humphreys, J.C. Gates, H.L. Rogers, C. Söller, B.J. Smith, W.S. Kolthammer, P.G.R. Smith et al., Optica 4, 90 (2017)

[8] V. Ansari, E. Roccia, M. Santandrea, M. Doostdar, C. Eigner, L. Padberg, I. Gianani, M. Sbroscia, J.M. Donohue, L. Mancino et al., Opt. Express 26, 2764 (2018)

[9] C. Joshi, A. Farsi, S. Clemmen, S. Ramelow, A.L. Gaeta, Nat. Commun. 9, 847 (2018)

[10] C.K. Law, I.A. Walmsley, J.H. Eberly, Phys. Rev. Lett. 84, 5304 (2000)

[11] D.B. Horoshko, G. Patera, A. Gatti, M.I. Kolobov, Eur. Phys. J. D 66, 239 (2012)

[12] S.M. Barnett, P.M. Radmore, Methods in theoretical quantum optics (Clarendon Press, Oxford, 1997)

[13] M.I. Kolobov, Rev. Mod. Phys. 71, 1539 (1999) 
[14] U.M. Titulaer, R.J. Glauber, Phys. Rev. 140, B676 (1965)

[15] C.T. Lee, Phys. Rev. A 52, 3374 (1995)

[16] C. Navarrete-Benlloch, An introduction to the formalism of quantum information with continuous variables (Morgan and Claypool Publishers, 2015)

[17] D. Hogg, D.W. Berry, A.I. Lvovsky, Phys. Rev. A 90, 053846 (2014)

[18] G.S. Agarwal, K. Tara, Phys. Rev. A 46, 485 (1992)

[19] T. Kiesel, W. Vogel, V. Parigi, A. Zavatta, M. Bellini, Phys. Rev. A 78, 021804 (2008)

[20] B. Yurke, D. Stoler, Phys. Rev. Lett. 57, 13 (1986)

[21] D.B. Horoshko, S.Y. Kilin, Opt. Express 2, 347 (1998)

[22] D.B. Horoshko, S.Y. Kilin, J. Exp. Theor. Phys. 90, 733 (2000)

[23] S. Haroche, J.M. Raimond, Exploring the Quantum: Atoms, Cavities and Photons (Oxford University Press, 2006)

[24] D.B. Horoshko, S. De Bièvre, M.I. Kolobov, G. Patera, Phys. Rev. A 93, 062323 (2016)

[25] C.W. Lee, H. Jeong, Phys. Rev. Lett. 106, 220401 (2011)

[26] B. Yadin, V. Vedral, Phys. Rev. A 93, 022122 (2016)

[27] S.D. Bièvre, D.B. Horoshko, G. Patera, M.I. Kolobov, arXiv: 1809.02047 